\title{
New perspectives on catecholaminergic regulation of executive circuits: evidence for independent modulation of prefrontal functions by midbrain dopaminergic and noradrenergic neurons
}

\author{
Daniel J. Chandler, Barry D. Waterhouse and Wen-Jun Gao* \\ Department of Neurobiology and Anatomy, Drexel University College of Medicine, Philadelphia, PA, USA
}

\section{Edited by:}

Evelyn K. Lambe, University of

Toronto, Canada

\section{Reviewed by:}

Rita J. Valentino, The Children's Hospital of Philadelphia, USA

Amy F. T. Arnsten, Yale University

School of Medicine, USA

\section{*Correspondence:}

Wen-Jun Gao, Department of Neurobiology and Anatomy, Drexel University College of Medicine, 2900 Queen Lane, Philadelphia, PA 19129, USA

e-mail:wgao@drexelmed.edu

\begin{abstract}
Cognitive functions associated with prefrontal cortex (PFC), such as working memory and attention, are strongly influenced by catecholamine [dopamine (DA) and norepinephrine $(\mathrm{NE})]$ release. Midbrain dopaminergic neurons in the ventral tegmental area and noradrenergic neurons in the locus coeruleus are major sources of DA and NE to the PFC. It is traditionally believed that DA and NE neurons are homogeneous with highly divergent axons innervating multiple terminal fields and once released, DA and NE individually or complementarily modulate the prefrontal functions and other brain regions. However, recent studies indicate that both DA and NE neurons in the mammalian brain are heterogeneous with a great degree of diversity, including their developmental lineages, molecular phenotypes, projection targets, afferent inputs, synaptic connectivity, physiological properties, and behavioral functions. These diverse characteristics could potentially endow DA and NE neurons with distinct roles in executive function, and alterations in their responses to genetic and epigenetic risk factors during development may contribute to distinct phenotypic and functional changes in disease states. In this review of recent literature, we discuss how these advances in DA and NE neurons change our thinking of catecholamine influences in cognitive functions in the brain, especially functions related to PFC. We review how the projection-target specific populations of neurons in these two systems execute their functions in both normal and abnormal conditions. Additionally, we explore what open questions remain and suggest where future research needs to move in order to provide a novel insight into the cause of neuropsychiatric disorders related to DA and NE systems.
\end{abstract}

Keywords: catecholamine, dopamine, norepinephrine, prefrontal cortex, executive function

\section{INTRODUCTION}

The prefrontal cortex (PFC) is involved in a number of cognitive and executive functions in both primates and rodents, including working memory, sustained and flexible attention (Dalley etal., 2004; Arnsten, 2009; Bari and Robbins, 2013), and is therefore critical in guiding behavior in a complex and dynamic world. Importantly, PFC is innervated and strongly modulated by a number of anatomically and neurochemically distinct pathways. Of particular interest are the afferent fibers arising in the dopaminergic ventral tegmental area (VTA) and noradrenergic locus coeruleus (LC). The anatomical characteristics of these two catecholamine nuclei, as well as the cellular, physiological, and behavioral consequences of their activation, have been well characterized and reviewed in the past dopamine [DA - (Seamans and Yang, 2004; Bjorklund and Dunnett, 2007; Grace et al., 2007; Schultz, 2007; Bromberg-Martin et al., 2010; Ungless and Grace, 2012; Roeper, 2013), norepinephrine (NE) - (Dahlstroem and Fuxe, 1964; Morrison et al., 1978; Grzanna and Molliver, 1980; Swanson, 1982; Berridge and Waterhouse, 2003; Devilbiss and Berridge, 2006; Devilbiss etal., 2006; Arnsten, 2007; Chandler and Waterhouse, 2012; Chandler et al., 2013)]. It is important to note that these two systems vary to a degree between rodents and primates. In particular, DA fibers in the primate PFC are known to arise from both the substantia nigra and VTA (Porrino and Goldman-Rakic, 1982; Haber and Fudge, 1997). In addition, in contrast to the popular view that DA-containing fibers project selectively to PFC in primates, the heaviest cortical DA projection actually terminates in motor and premotor cortices in the primate brain, while there seems to be a preferential DA projection to frontal and temporal areas in the rat with a minimal contribution to primary sensory and motor areas (Lewis et al., 1987; Berger et al., 1991). Furthermore, the distribution of DA-containing fibers among the cortical layers differs between species such that in primates, layer I is most densely innervated throughout the majority of the cortical mantle, whereas layers I through III are most densely innervated in the rat, and that this occurs preferentially in cingulate and entorhinal cortices (Berger et al., 1991). Despite the inter-species differences in DA projections to cortex [for more detailed review, see (Berger et al., 1991; Haber and Fudge, 1997)], we will focus 
on recent findings describing the functional organization and neuronal diversity within VTA and LC and how these attributes relate to the execution of distinct behaviors maintained by prefrontal and non-prefrontal neural circuits. We will also consider how these two systems act synergistically within their terminal fields to mutually guide several aspects of complex behaviors. Finally, although there have been many more recent breakthroughs in understanding dopaminergic neuromodulation of prefrontal circuits, we will discuss how these advances can serve as a guide to similarly transform our thinking about the LC-PFC pathway.

\section{DIVERSE FUNCTIONS AND PROPERTIES OF VTA DA NEURONS}

It is well established that the VTA includes both DA and non-DA neurons which project heavily to both PFC and the nucleus accumbens (NAc; Swanson, 1982; Lammel et al., 2008). This projection system has been strongly linked to normal cognitive function and motivated behavior, as well as pathological deviations in these operations such as schizophrenia, attention deficit hyperactivity disorder (ADHD) and addiction (Goldman-Rakic, 1994; Goldman-Rakic and Selemon, 1997; Volkow et al., 2011, 2012). In both rodents and primates, the actions of prefrontal cortical DA are known to vary according to an "inverted U" dose response function, such that too little or too much DA impairs PFC network functions and working memory task performance (Arnsten and Goldman-Rakic, 1998; Arnsten and Li, 2005; Robbins and Arnsten, 2009). It is also known that the firing properties of VTA dopaminergic neurons are plastic such that they are capable of remaining in a silent hyperpolarized state, maintaining irregular tonic discharge, and firing phasically in response to environmental stimuli under different behavioral conditions. However, because DA seems to execute distinct operations in different terminal fields (i.e., reward and reinforcement in NAc and enhancement of working memory in PFC), it raises the question of whether or not the cells which provide DAergic innervations to these regions are anatomically distinct from one another, and whether or not these cells can be differentially activated under different circumstances. Indeed, previous studies have provided evidence for functional specialization of mesocortical DA neurons (Bannon et al., 1982; Chiodo et al., 1984). This issue was recently further illustrated and detailed by Lammel et al. (2008) who found that in rodent, PFC and NAc are in fact innervated by distinct subsets of VTA neurons, and that these cells are physiologically and phenotypically distinct from one another. Specifically, the neurons that project to NAc were found to discharge slowly and have their firing rate suppressed by application of DA, whereas those that project to PFC discharged more rapidly and did not respond to DA application. These discharge properties can be explained by the fact that PFC projection cells lack mRNA coding for the DA D2 autoreceptor, which inhibits firing of DA neurons. Taken together these findings suggest that NAc and PFC which are engaged in unique aspects of motivated behavior receive input from anatomically distinct subsets of DA containing neurons, whose firing patterns appear to be under differential control.

A follow-up to this study showed that each of these subsets of DA-containing neurons in VTA are likewise unique in their afferent regulation, and that these distinct circuits support different types of behaviors. Specifically, it was shown that afferents from the laterodorsal tegmental nucleus innervate dopaminergic VTA neurons which in turn project to NAc and elicit reward, whereas afferents from the lateral habenula ( $\mathrm{LHb}$ ) synapse on dopaminergic VTA neurons that innervate mPFC and drive aversion (Figure 1). The conclusion from this work is that the VTA is comprised of neurochemically similar but anatomically and functionally distinct neurons that mediate discrete aspects of motivated behaviors (Lammel et al., 2012). It is interesting to note that activation of dopaminergic neurons with projections to $\mathrm{mPFC}$ results in conditioned place aversion, whereas a much greater body of literature suggests that DA in the PFC plays an important role in electrophysiological and behavioral indices of working memory (Goldman-Rakic, 1994; Stevens et al., 1998; Goldman-Rakic et al., 2004; Arnsten and Li, 2005; Arnsten, 2007; Driesen et al., 2008).

These dual roles for DA in the PFC could potentially be explained by the existence of anatomically and functionally discrete subsets of VTA DA neurons that innervate different cortical layers: for example, DA neurons involved in working memory may project primarily to cortical layers that interact with primary sensory cortices to facilitate the transmission of sensory information between regions so that the representation of a stimulus can be maintained even in its absence. Aversion and emotional operations maintained by DA, on the other hand, may involve the activation of DA neurons that innervate cortical layers which maintain connections with limbic structures rather than sensory structures. In this way, activation of these two pathways could result in DA release and modulation of functionally distinct prefrontal microcircuits that mediate unique operations and behaviors. Conversely, these unique functions could be attributed to a common pool of VTA neurons that do not selectively target functionally distinct cortical layers but, depending on their pattern and level of activation, engage different receptor subtypes to elicit distinct circuit properties. For example, during modest levels of VTA output, such as in response to salient stimuli, the D2 receptor will be activitated due to its higher affinity for the transmitter. Then, during elevated levels of VTA activation, such as during periods of stress, the lower affinity D1 receptor becomes engaged. Thus, because of different receptor affinities and post-synaptic actions, DA release would produce different effects on cellular physiology and PFC circuit properties (Arnsten, 2007, 2009). Based on the inverted-U dose response function for DA actions, and the differential roles of its receptors in working memory functions, modest DA release in response to a salient stimulus is likely to strengthen measures of working memory for that stimulus, whereas excessive activation of the DA D1 receptor impairs behavioral indices of working memory (Seamans and Yang, 2004; Arnsten, 2009). During such periods when PFC is inhibited, emotional centers such as amygdala may instead take over and drive more survivalist "fight or flight" behaviors (Arnsten, 2009). In such an organization, the aversion described by Lammel et al. (2012) may have been reflective of hyperdopaminergic tone at the upper limit of the physiologic range in PFC as a result of optogenetic stimulation, thereby limiting prefrontal operations and allowing other 


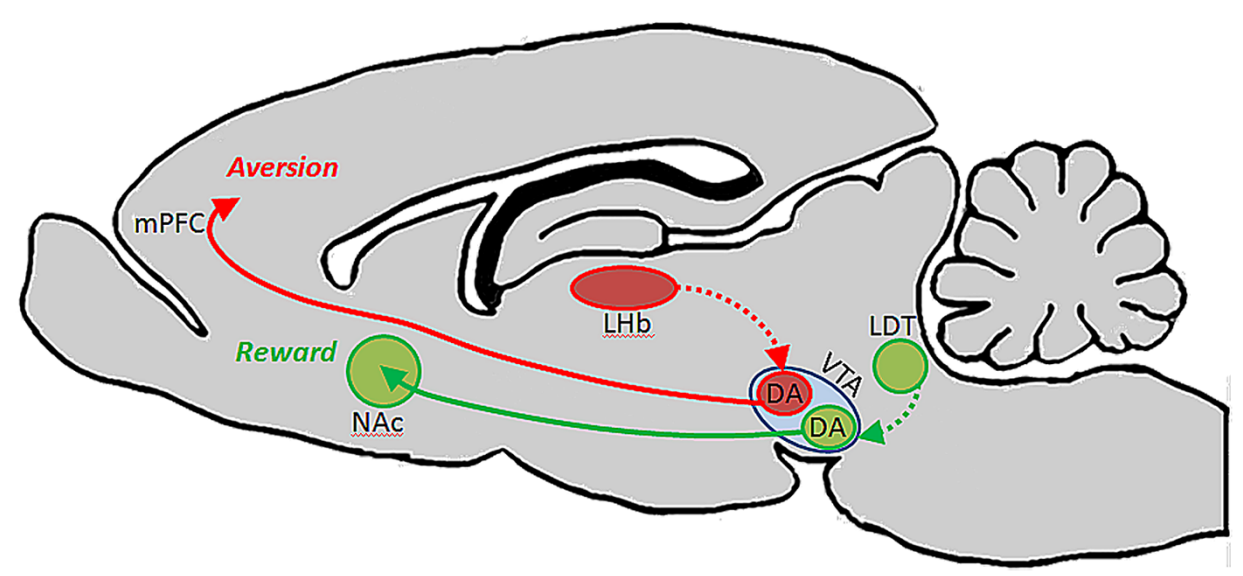

FIGURE 1 |Ventral tegmental area contains functionally heterogeneous subsets of DA neurons. Lammel etal. (2012) showed that DA cells in VTA which receive input from laterodorsal tegmentum (LDT) selectively project to NAc, and their activation drives conditioned place preference. DA cells in VTA which receive input from lateral habenula ( $\mathrm{LHb}$ ) neurons, on the other hand, project selectively to PFC, and their activation promotes conditioned place aversion. limbic circuits to guide such a specific behavior instead. Interestingly, Bromberg-Martin et al. (2010) has hypothesized that in the primate brain, DA cells arising from substantia nigra and VTA differentially innervate orbitofrontal cortex and dorsolateral PFC to convey value and salience, respectively, to these structures. This proposal fits well with our central hypothesis that specific subpopulations of neurons arising from the midbrain and hindbrain nuclei are capable of executing unique actions in distinct terminal fields.

\section{DIVERSITY OF NE NEURONS IN THE LC NUCLEUS}

Prefrontal circuits and operations are also subject to regulation by output from the LC-noradrenergic system. Like DA, the actions of $\mathrm{NE}$ vary according to an inverted-U dose response function such that too little or too much noradrenergic transmission yields a less than optimal neuronal response to sensory stimuli (Berridge and Waterhouse, 2003; Devilbiss and Waterhouse, 2004; Devilbiss et al., 2012). Importantly, the pattern of LC activation correlates highly with behavioral state in both primates and rodents such that during periods of fatigue, LC discharge is absent or slow. During periods of active waking and in conjunction with behavioral tasks that are cognitively demanding, the LC discharges faster with phasic bursts in response to relevant stimuli. During periods of stress and agitation, the nucleus discharges at a very high tonic rate and sensory-driven phasic responses are lost (Aston-Jones and Bloom, 1981a; Valentino and Foote, 1988; Aston-Jones et al., 1994; Berridge and Waterhouse, 2003; Aston-Jones and Cohen, 2005a,b). Likewise, too much NE in PFC synapses activates the $\alpha 1$ receptor, impairing PFC function in a manner similar to excessive activation of the D1 receptor (Arnsten and Dudley, 2005; Arnsten, 2007, 2009).

Interestingly, behavioral and electrophysiological studies of LC in both primate (Aston-Jones et al., 1994) and rodent (Bouret and Sara, 2004) have shown that LC is highly plastic in response to stimuli that drive its activation. Previous work had suggested a more simplistic role for the LC-NE system in arousal and the sleep-waking cycle. However, attended stimuli that predict reward have been found to elicit a robust phasic discharge of LC cells, while distracters of the same or different modality do not (Aston-Jones et al., 1994). Importantly, the response to a reward-predicting stimulus is rapidly lost and shifted to a new stimulus when the reward-contingency is changed (Foote et al., 1980; Aston-Jones et al., 1994; Rajkowski et al., 1994; Bouret and Sara, 2004; AstonJones and Cohen, 2005a). These data suggest that LC may therefore have a more complex role in attention and cognition, than simply serving as a generalized alerting or wake-promoting structure (Aston-Jones and Bloom, 1981a; Rajkowski et al., 1994; Berridge and Waterhouse, 2003; Berridge, 2008). Aston-Jones and Cohen, for example, have proposed that LC integrates goal-oriented sensory information from the PFC to shift the nucleus between tonic and stimulus-driven phasic modes of discharge. These tonic and phasic modes of discharge then sensitize terminal fields to detect non-specific and specific stimuli, respectively; thereby guiding labile versus sustained modes of attention (Aston-Jones and Cohen, 2005b).

Importantly, it has long been thought that LC is the sole source of NE to the neocortex (Loughlin etal., 1982, 1986a,b; Berridge and Waterhouse, 2003; Agster etal., 2013), and that its' neurons project to their terminal fields indiscriminately; i.e., a single neuron is just as likely to innervate functionally dissimilar regions as those that have common function (Loughlin et al., 1982, 1986a,b). Recent behavioral evidence however, seems to suggest that the LC-NE system exerts unique influences on operations in distinct prefrontal terminal fields. Specifically, in rodent, NE specific lesions of mPFC impair extradimensional shifting, a behavior in which animals must reorient their attentional reserves to novel stimuli to obtain food reward, but not reversal learning, an OFC dependent behavior in which animals must reorient attention to familiar but previously irrelevant stimuli (McGaughy et al., 2008b; Newman et al., 2008). On the basis of these findings and the observation that both behaviors are noradrenergically regulated (McGaughy et al., 2008a,b; Seu et al., 
2009; Snyder et al., 2012) we postulated that OFC and mPFC must be innervated by distinct subsets of LC neurons: if both regions received input from a common pool of LC neurons, injection of 6-OHDA into $\mathrm{mPFC}$ would lead to the retrograde degeneration of the axons in mPFC, the cell bodies in LC, as well as anterograde degeneration of axon collaterals innervating OFC. Indeed, we have recently shown that these two regions, as well as anterior cingulate cortex, a third anatomically and functionally distinct prefrontal region, are in fact innervated by anatomically distinct subsets of LC neurons (Chandler and Waterhouse, 2012; Chandler et al., 2013). Additionally, another recent publication from our laboratory demonstrated that the density of noradrenergic release points is not uniform throughout the forebrain (Agster et al., 2013). Specifically, NE varicosity is significantly more dense in PFC than in sensory, motor, and thalamic regions, further supporting the hypothesis that NE may have unique roles and execute distinct operations in functionally and anatomically disparate projection fields (Figure 2). These findings suggest that the LC-NE projection to PFC subregions may subserve distinct behavioral roles, similar to what is suggested by the organization of the mesolimbic and mesocortical dopaminergic pathways described by Lammel et al. (2008, 2012). It has also recently been demonstrated by Robertson et al. (2013) that contrary to the longheld notion that LC is the sole source of NE-containing fibers to the forebrain in rodents, the insular cortex is innervated by non-LC derived NE terminals, i.e., sub-coeruleus, A1, and A2 cell groups (Figure 2). Such findings challenge the classical view that NE acts uniformly and synchronously within its terminal fields (Aston-Jones and Bloom, 1981a,b; Rajkowski et al., 1994). Specifically, NE release in insular cortex may be achieved through activation of LC, or by activation of the functionally and anatomically distinct sub-coeruleus, A1, or A2 cell groups. The different anatomical connectivities and physiological attributes of these various noradrenergic nuclei suggest that NE can be released into
PFC under unique sensory or environmental circumstances. The finding that PFC is the only cortical structure in this study to be innervated by non-LC NE fibers suggests that the transmitter may maintain unique roles in prefrontal versus non-prefrontal cortical function. Because these non-LC noradrenergic cell groups receive sensory information from the viscera and are involved in homeostatic and interoceptive functions, they form an autonomic circuit and a direct route for the release of NE into prefrontal structures that affect vigilance and decision making. This pathway bypasses the LC and provides a means for asynchronous release of NE in the forebrain from multiple brainstem structures. Such an organization would therefore impose changes in prefrontal physiology without affecting properties of other terminal fields and argues that NE discretely modulates anatomically and functionally distinct terminal networks. Such a hypothesis could be tested by electrically or optogenetically stimulating these non-coerulear noradrenergic cell groups while sampling NE release in prefrontal versus non-prefrontal terminal fields by microdialysis or fast scan voltammetry.

\section{RECIPROCAL CONNECTIONS BETWEEN LC AND VTA}

Despite the heterogeneous and varied roles for DA and NE in prefrontal cortical function that have already been discussed, an added layer of complexity emerges when taking into account the reciprocal connections maintained between VTA and LC (El Mansari et al., 2010). It is reasonable to expect that as both of these pathways are activated in response to different behavioral circumstances, each will produce some effect on the other. This then begs the question as to whether these systems work cooperatively to produce behavioral modifications that require output from both systems, or if they act competitively to drive distinct and opposite behavioral outcomes. It has been shown that electrical stimulation of LC results in an excitation followed by a brief inhibition of midbrain dopamine (DA) neurons through an

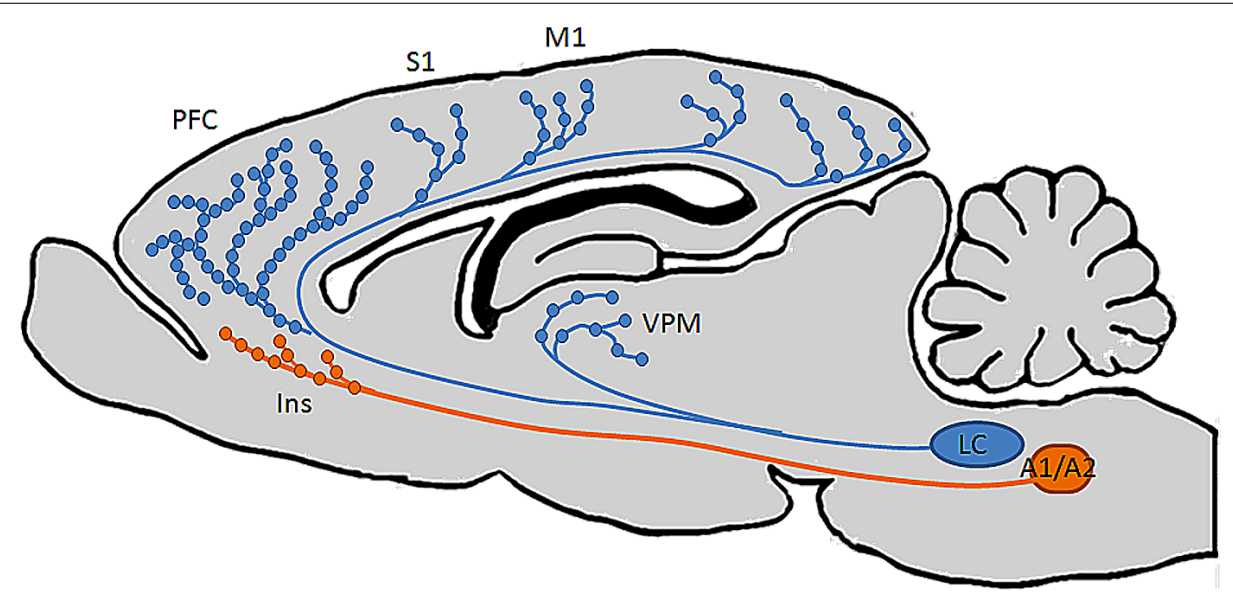

FIGURE 2 | Distinct brain regions are differentially innervated by noradrenergic neurons in multiple brainstem nuclei. Recent findings from our laboratory (Chandler and Waterhouse, 2012; Agster etal., 2013; Chandler etal., 2013) show that individual LC neurons innervate multiple functionally distinct cortical terminal fields, and that the highest density of NE varicosities in the brain occurs in PFC. A recent finding by Robertson etal. (2013) also challenged the longstanding notion that LC is the sole source of NE to cortex by demonstrating the existence of NE-containing fibers in insular cortex derived from a rhombomere distinct from that in which LC develops, suggesting that this region has privileged access to autonomic and visceral information while the rest of the cortical mantle does not. 
$\alpha 1$ receptor dependent mechanism (Grenhoff et al., 1993). Furthermore, lesions of LC have been shown to reduce basal and amphetamine-induced release of DA in the NAc (Lategan et al., 1990). Interestingly, anatomical evidence has shown that there is also a monosynaptic projection from VTA to LC (Beckstead et al., 1979), and that stimulation of VTA increases the concentration of NE metabolites in PFC (Deutch et al., 1986). Furthermore, previous studies indicated that both NE and DA provide essential modulatory influences on prefrontal functions (Mingote et al., 2004; Arnsten and Li, 2005; Aston-Jones and Cohen, 2005b; Morilak et al., 2005; Rossetti and Carboni, 2005; Drouin et al., 2006).

How do these two systems coordinate their activities to appropriately regulate prefrontal functions and what happens when this coordination becomes un-balanced? Essentially, how does one system affect the ouput of the other under normal conditions and disease states? DA and NE are critical for maintaining normal, adaptive behaviors (Arnsten and Goldman-Rakic, 1998; Dalley et al., 2004; Aston-Jones and Cohen, 2005a; Arnsten, 2007; McGaughy et al., 2008a,b). Increasing or decreasing either transmitter severely limits exploratory behavior. VTA and LC neurons that release DA and NE, respectively, are both activated by salient stimuli, and the strength of activation appears to be related to the values of stimuli used for predicting future behavior (Horvitz, 2000; Stuber et al., 2008; Sara and Bouret, 2012). However, existing evidence suggests DA and NE may contribute to different functions, with DA being related to reward assessment and error prediction and NE being related to arousal and/or vigilance. This suggests that their roles in motivated behavior are segregated in that they reflect different influences of reward on behavior. It has been postulated that DA neurons are more sensitive to the incentive value of reward information, whereas NE neurons are more sensitive to the arousing aspects of reward information (Bouret et al., 2012). Similarly, during a working memory task, NE and DA systems also synergistically or complementarily contribute to modulate the persistent activity needed for the cue, delay and response signaling within the PFC circuitry. Specifically, as others (Robbins and Arnsten, 2009; Arnsten, 2009) have proposed, with optimal levels of NE or DA release under alert, non-stressed conditions, PFC neurons fire during the delay period following cues for preferred but not non-preferred directions. NE enhances delay-related firing in response to cues in preferred directions by stimulating $\alpha 2 \mathrm{~A}$-receptors (increasing the "signal"), whereas DA weakens delay-related firing in response to cues in non-preferred directions by stimulating D1 receptors (decreasing the "noise"). This assumption is evidenced by administration of appropriate concentrations of the $\alpha 2 \mathrm{~A}$-receptor agonist guanfacine or the D1 receptor agonist SKF81297. In contrast, with high levels of $\mathrm{NE}$ and DA release as would occur during stress, NE engages the lower-affinity $\alpha 1$-receptors and reduces mnemonic stimulus evoked neuronal firing. Interestingly, the impact of the activation of adrenergic receptors in non-prefrontal cortical regions such as sensory and motor cortices seem to be opposite of that in prefrontal regions: $\alpha 1$-receptor activation increasees neuronal responsiveness to sensory-driven inputs, whereas $\alpha 2$ receptor activation suppresses stimulus evoked discharge (Arnsten, 2000, 2007, 2009). Similarly, high DA induces excessive D1 receptor stimulation and suppresses cell firing as well. Indeed, administration of the $\alpha 1$-receptor agonist phenylephrine (Mao et al., 1999) or a high concentration of SKF81297 (Williams and GoldmanRakic, 1995) can mimick the effects of high NE and DA levels, respectively.

It is also important to recognize that DA and NE levels in PFC are constantly fluctuating as a function of arousal level and ongoing behavioral contingencies. As the relative levels of these transmitters in the extracellular space changes, so too will their impact on cellular function. Importantly, the impact of these transmitter systems on post-synaptic cellular physiology is often characterized one at a time, i.e., the impact of DA or the impact of NE on specific parameters of neuronal or circuit function. However, under physiological conditions, it is likely that these two transmitters, as well as many other neuromodulatory agents and transmitter substances interact simultaneously throughout the brain and spinal cord via activation of a number of membranebound receptors on neurons and glia. A first step in addressing the issue of neuromodulator interactions and influences on complex circuit functions would be to consider the net effects of simultaneous administration of two or more modulatory substances on synaptically driven discharge of target neurons. There is already strong evidence for synapse- and cell-type specific modulation of local cortical circuitry in the PFC by both DA and NE (Gao et al., 2001, 2003; Gao and Goldman-Rakic, 2003; Wang et al., 2013). Thus, the PFC is a likely candidate for studies focused on the combined impact of DA and NE on transmission at single synapses and response properties of identified neurons.

\section{SUMMARY}

Taken together the findings reviewed here suggest that both noradrenergic and dopaminergic nuclei contain heterogeneous sets of neurons whose properties vary according to terminal field projection targets, and that these two catecholamine pathways act synergistically or complementarily in order to affect executive function and motivated behaviors via connections with specified forebrain circuits as well as by maintaining reciprocal excitatory connections with one another. Because there exists a range of concentrations for both DA and NE in PFC at which behavior and cellular physiology are optimized, and too far below or above this range is detrimental to behavioral outcomes, it seems that these two systems are both required for the normal maintenance and execution of prefrontal operations. Likewise, because these two pathways are reciprocally excitatory, it is likely that activation of one pathway by external or internal stimuli recruits the other indirectly. Such an arrangement would benefit complex behaviors, i.e., a task requiring sustained attention is also dependent on motivational state. It may be the case that VTA efferents to NAc and PFC work in concert with LC inputs to PFC and primary sensory and motor cortical regions. For example, during a period of vigilance in a particular behavioral task, LC activation and NE release may optimize PFC and sensory cortical function with respect to signal to noise ratios of stimulus evoked pyramidal neuron responses, while DA release from VTA promotes a transient working memory association - mnemonic - of that stimulus. Together, these two transmitter systems work synergistically to allow the animal to selectively focus on and remember 
the relevance of a reward associated stimulus. Upon the successful execution of a behavioral trial and reward retrieval, VTA signals NAc to elicit reward, reinforcing the behavior and causing the animal to continue focusing on that specific stimulus to predict and retrieve the next reward. Hereafter, when a behavioral contingency is changed, the NAc signals VTA that an expected reward has not occurred. The reciprocal connections between VTA and LC may then alter their collective output in PFC, thereby decreasing the sensitivity of PFC and primary sensory networks to that specific stimulus by a NE-mediated decrease in signal to noise ratio, as well as a decrease in working memory for that stimulus. Consequently, in the absence of reinforcement and reward, the animal is able to sample alternative behavioral strategies through sensitization to previously irrelevant stimuli. Once a new strategy is identified, VTA signals NAc to promote reward, thereby shifting the reciprocal connections between LC and VTA back to a mode which favors sensory discrimination and working memory of the new reward predictive stimulus (Figure 3). This is an intriguing possibility given that in the rodent, NAc and striatum seem to be largely devoid of LC-derived fibers, and primary sensory and motor cortical areas are not heavily innervated by DA fibers (Berger et al., 1991; Berridge and Waterhouse, 2003). Hence, VTA may preferentially modulate reward through its projections to NAc, LC may preferentially modulate sensory and motor processes through its projections to more posterior cortical areas, and these two catecholamine nuclei may work synergistally in PFC to affect attention, working memory, and cognitive functions that drive complex behavior (Figure 4).

Therefore, reciprocal connections between these two nuclei may be important for maintaining activity states in each nucleus that are sufficient for appropriately guiding ongoing behavior. In the absence of these reciprocal connections, the projection from VTA to NAc might be sufficient for keeping an animal motivated to perform a task or execute a specific behavior, but attention toward a specific stimulus used to guide that behavior may be minimal. Conversely, the projection from LC to PFC might be adequate in resolving specific stimuli, but insufficient to attend specific stimuli and achieve a desirable outcome in the absence of a motivational drive provided by the dopaminergic projection from VTA to NAc.

Additionally, as discussed earlier, the VTA maintains a projection to PFC which has been shown to promote aversion (Lammel et al., 2012) rather than motivation or reward. Interestingly, it is known that certain stressors elicit greater release and metabolism of DA in PFC than other forebrain regions (Deutch and Roth, 1990), suggesting that the mesocortical DA may play an integral role in the cognitive aspects of the stress response. Importantly, it is also known that high levels of DA and NE in PFC impair cognition and elevation of these catecholamines occur during exposure to stressors. During stressor-induced activation of the LC (Valentino and Foote, 1987, 1988; Curtis et al., 1999; Berridge and Waterhouse, 2003; Berridge, 2008; Devilbiss et al., 2012), the VTA would be the target of increased noradrenergic transmission from the LCVTA pathway, thereby providing a means for VTA to contribute to the expression of aversive behaviors much in the same way that $\mathrm{LHb}$ neurons influence VTA activity and DA release within the PFC (Lammel et al., 2012, 2013). Methods similar to those used by Lammel et al. (2012) could be employed to identify such functional connections between VTA and LC and to determine how the reciprocal connections between these two nuclei influence physiological properties, release, and consequently PFC related cognitive function and behavior.

Importantly, these recent findings on the neurobiology of the VTA, as well as the recent identification of non-LC derived NEcontaining terminals in insular cortex represent a way forward for advancing the study of the LC-NE pathway. As this system has long been viewed as homogeneous with fairly uniform, synchronous actions across its efferent domain and on behavior by way of a highly divergent network of axon collaterals, the demonstration that it is in fact more heterogeneous than previously recognized would transform the prevailing notions about the postulated contributions of the LC-NE system to forebrain operations. Importantly, we have recently provided anatomical evidence that LC neurons innervate their terminal fields on a functional rather than random basis (Chandler and Waterhouse, 2012; Chandler et al., 2013) and experiments are currently underway to test the hypothesis that cells with discrete terminal fields express different molecular profiles and unique physiological attributes. Such data would provide evidence that LC efferent system is capable of differential release and asynchronous $\mathrm{NE}$ actions across its terminal fields in the same way that DA release is governed by specified VTA projection patterns. Additionally, the recent demonstration that certain regions of PFC are innervated by nonLC-NE containing fibers (Robertson et al., 2013) supports the view that NE maintains distinctive roles in prefrontal circuit operations as dictated by activation of source nuclei (sub-coeruleus, A1, A2) that give rise to NE-PFC projections. Such an organization would therefore prompt noradrenergic modulatory actions in prefrontal circuits without affecting other cortical regions; a mode of operation similar to that proposed for the VTA-DA system on the basis of its divergent mesocortical and mesolimbic projections.

Identification of specific afferents to LC cells with specified outputs as has been shown in the DA system (Lammel et al., 2008, 2012) will further the collective understanding of the role of LC in maintaining discrete behavioral operations rather than acting as a homogeneous and uniform modulator of the activity in LC projection fields. Optogenetic approaches may provide a means of characterizing anatomic, neurochemical, and functionally specific pathways into and out of LC that maintain distinct roles and demonstrate that NE release is capable of producing unique actions in different terminal fields under diverse circumstances. Because the LC-NE and VTA-DA systems maintain reciprocal anatomical connections and appear to act synergistically and complementarily to guide behavior, advances in the study of one of these catecholamine pathways will by necessity impact study of the other. Going forward it will be important to consider the differences as well as the similarities between these two systems. Nevertheless, the results of recent studies of the VTA show that heterogeneity is quite apparent in the nucleus (Lammel et al., 2008, 2012), and our recent work on the anatomy of the LCPFC projections show that the nucleus is at least anatomically aligned to allow for similar heterogeneity in this nucleus as well. As such, anatomical, molecular, and physiological heterogeneity 

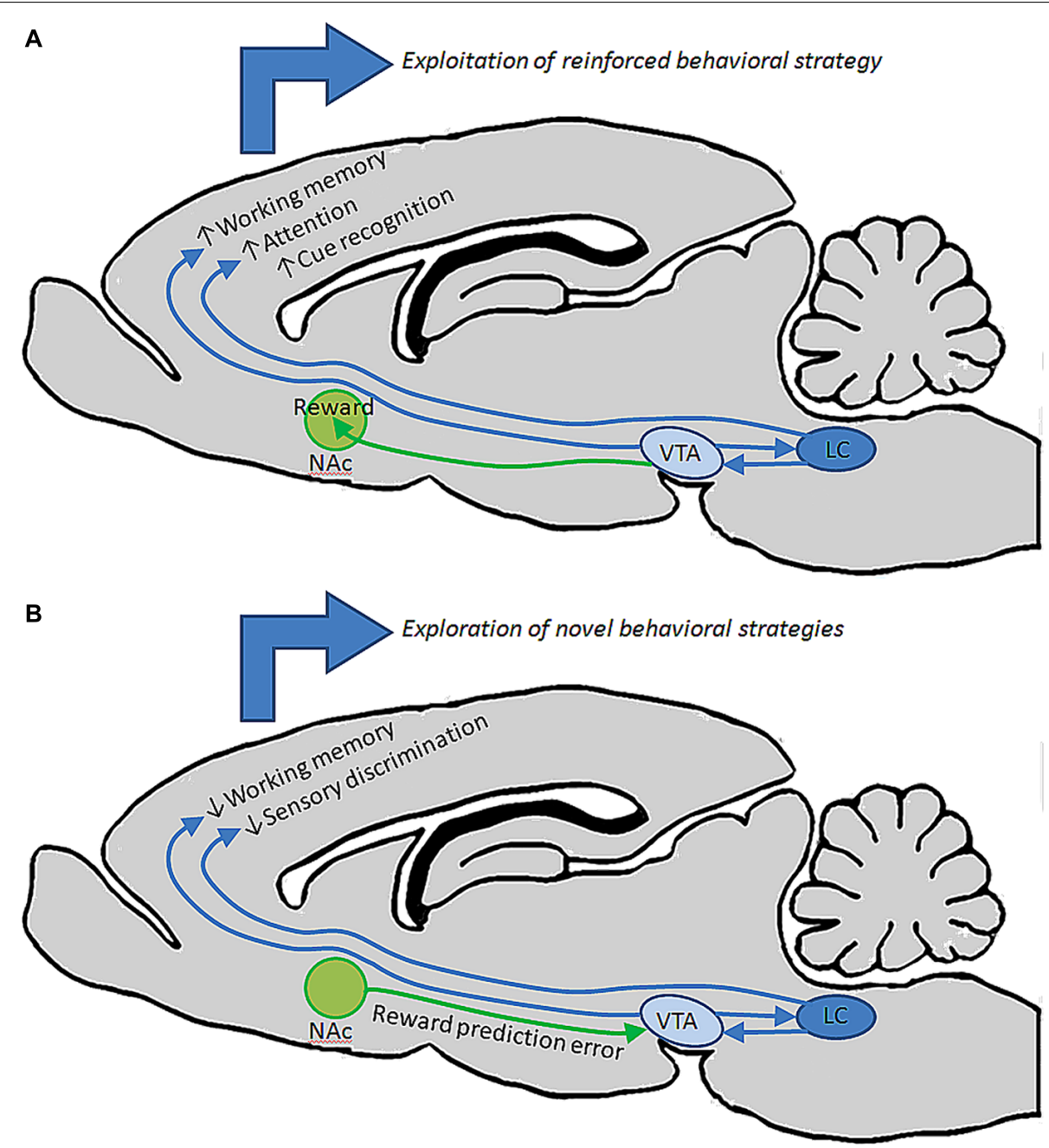

FIGURE 3 | Ventral tegmental area and LC may work together to guide behavior under different circumstances. (A) During performance in a behavioral task in which an animal is successfully retrieving reward, reciprocal connections between VTA and LC may facilitate elevated output from these nuclei, driving release of DA and NE in PFC to promote working memory, attention, and discrimination of specific stimuli that predict reward. These behavioral operations could collectively contribute to the repetition of that behavior until reward is retrieved. (B) When a previously successful behavioral strategy loses its relevance, NAc may signal to VTA that an expected reward has not occurred. Changes in VTA output could then alter LC output, collectively changing the level of DA and NE release in PFC to diminish working memory and discrimination of specific stimuli, instead allowing the animal to explore new behavioral strategies on the basis of detecting previously irrelevant stimuli. in catecholamine nuclei may therefore be a fundamental principle of their organization, and future studies of these structures and their efferent domains may provide a framework for better understanding acquired or genetically transmitted abnormalities of the VTA-DA and LC-NE systems that result in maladaptive behaviors including those expressed in addiction, ADHD, schizophrenia, and post-traumatic stress disorder.

\section{FUTURE PERSPECTIVE AND FUNCTIONAL IMPLICATIONS OF THE DIVERSITIES IN CATECHOLAMINERGIC INNERVATION OF PFC}

The diverse innervation of PFC by subsets of DA and NE neurons is certainly an important conceptual advance in our understanding of these two systems. But several questions remain. How are these two systems affected when PFC function and structure are altered in response to genetic and epigenetic factors? How do disease states affect each of these systems and their interactions? Are all cells within these nuclei equally responsive to genetic and environmental insult, or is it possible that cells with different terminal fields are differentially susceptible to certain forms of stressors? For example, evidence suggests that in Alzheimer's and Parksinson's diseases, LC neurons degenerate selectively (Gesi et al., 2000; Grimm et al., 2004; Weinshenker, 2008; Szot et al., 2010; McMillan et al., 2011; Miguelez et al., 2011). It may be that such degeneration targets LC-PFC projection neurons specifically and that this selective degeneration plays a role in the cognitive decline associated with these diseases. Further exploration of the properties of specified groups of LC-cortical projection neurons could help determine the susceptibility of these organizations to pharmacological, environmental, or genetic insult 


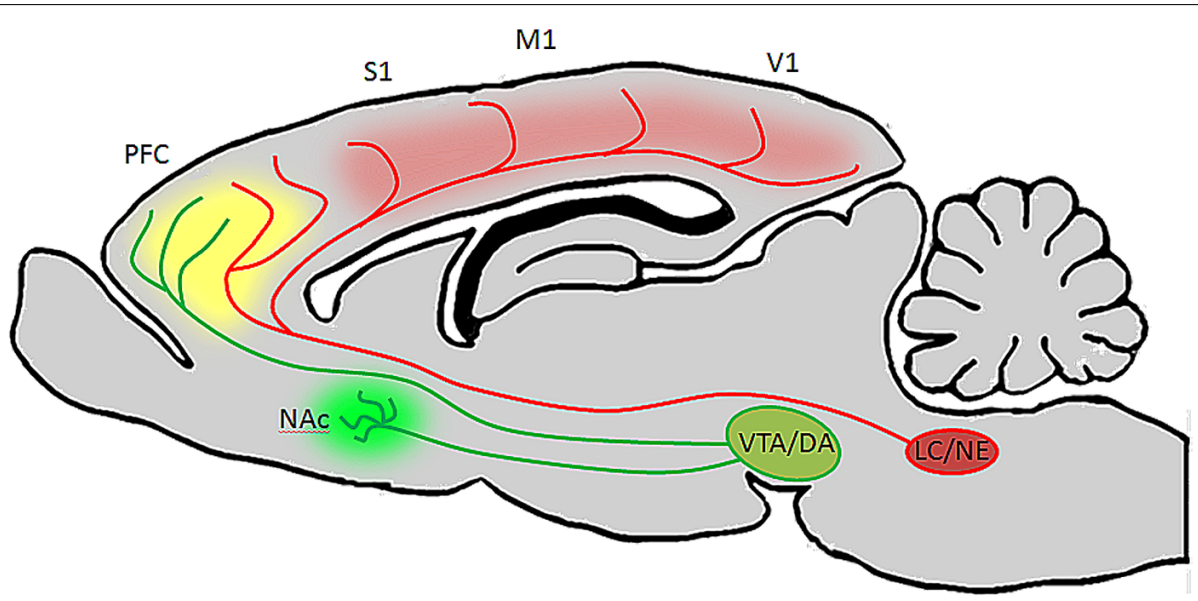

FIGURE 4 | Ventral tegmental area and LC neurons have distinct targets but their efferent fibers converge in PFC. In the rodent brain, LC projects heavily to the entire cortical mantle, including PFC and primary sensory and motor areas, but not to the striatum or NAc (Berridge and Waterhouse, 2003). VTA on the other hand innervates NAc and PFC, but provides only sparse innervations to more posterior cortical areas (Berger etal., 1991). Therefore, during periods of arousal and vigilance, when LC and VTA discharge is elevated, DA will be released in NAc, LC will be released in posterior cortical areas, and both catecholamines will be released in PFC. This may be beneficial during behavioral tasks which require sustained attention, as DA in NAc (green) will facilitate reward, NE in cortex (red) will alter the signal to noise ratio of pyramidal neurons to optomize them to specific stimuli, and both catecholamines in PFC (yellow) will work synergistically to facilitate working memory and attention to relevant stimuli. that manifest in symptoms of neuropsychiatric or neurodegenerative disease associated with noradrenergic function. Similarly, it remains to be determined whether PFC projection neurons in the LC are more sensitive to stressors or the actions of psychostimulant drugs (e.g., methylphenidate) as compared to LC cells with different efferent domains. Furthermore, based on the published data on VTA neurons, we expect that subtypes of LC neurons with unique profiles and terminal field projection patterns receive different sets of afferent inputs, e.g., GABAergic versus glutamatergic, cortical versus subcortical, as well as dopaminergic, serotoninergic, or cholinergic afferents. Answers to these questions will provide novel insights into the operation of these systems and their collective impact on adaptive and maladaptive behavior.

\section{ACKNOWLEDGMENTS}

This study was supported by NIDA DA017960 to Barry D. Waterhouse, Drexel HCEP fund to Daniel J. Chandler, Drexel Cure grant 002766-002 to Wen-Jun Gao and Barry D. Waterhouse, and NIH R01MH085666 to Wen-Jun Gao.

\section{REFERENCES}

Agster, K. L., Mejias-Aponte, C. A., Clark, B. D., and Waterhouse, B. D. (2013). Evidence for a regional specificity in the density and distribution of noradrenergic varicosities in rat cortex. J. Comp. Neurol. 521, 2195-2207. doi: 10.1002/cne. 23270

Arnsten, A. F. (2000). Through the looking glass: differential noradenergic modulation of prefrontal cortical function. Neural Plast. 7, 133-146. doi: 10.1155/NP.2000.133

Arnsten, A. F. (2007). Catecholamine and second messenger influences on prefrontal cortical networks of "representational knowledge": a rational bridge between genetics and the symptoms of mental illness. Cereb. Cortex 17(Suppl. 1), i6-i15. doi: $10.1093 /$ cercor/bhm033

Arnsten, A. F. (2009). Stress signalling pathways that impair prefrontal cortex structure and function. Nat. Rev. Neurosci. 10, 410-422. doi: 10.1038/ nrn 2648
Arnsten, A. F., and Dudley, A. G. (2005). Methylphenidate improves prefrontal cortical cognitive function through alpha2 adrenoceptor and dopamine D1 receptor actions: relevance to therapeutic effects in Attention Deficit Hyperactivity Disorder. Behav. Brain Funct. 1:2. doi: 10.1186/1744-9081-1-2

Arnsten, A. F., and Goldman-Rakic, P. S. (1998). Noise stress impairs prefrontal cortical cognitive function in monkeys: evidence for a hyperdopaminergic mechanism. Arch. Gen. Psychiatry 55, 362-368. doi: 10.1001/archpsyc.55. 4.362

Arnsten, A. F., and Li, B. M. (2005). Neurobiology of executive functions: catecholamine influences on prefrontal cortical functions. Biol. Psychiatry 57, 1377-1384. doi: 10.1016/j.biopsych.2004.08.019

Aston-Jones, G., and Bloom, F. E. (1981a). Activity of norepinephrine-containing locus coeruleus neurons in behaving rats anticipates fluctuations in the sleepwaking cycle. J. Neurosci. 1, 876-886.

Aston-Jones, G., and Bloom, F. E. (1981b). Norepinephrine-containing locus coeruleus neurons in behaving rats exhibit pronounced responses to non-noxious environmental stimuli. J. Neurosci. 1, 887-900.

Aston-Jones, G., and Cohen, J. D. (2005a). Adaptive gain and the role of the locus coeruleus-norepinephrine system in optimal performance. J. Comp. Neurol. 493, 99-110. doi: 10.1002/cne.20723

Aston-Jones, G., and Cohen, J. D. (2005b). An integrative theory of locus coeruleusnorepinephrine function: adaptive gain and optimal performance. Annu. Rev. Neurosci. 28, 403-450. doi: 10.1146/annurev.neuro.28.061604.135709

Aston-Jones, G., Rajkowski, J., Kubiak, P., and Alexinsky, T. (1994). Locus coeruleus neurons in monkey are selectively activated by attended cues in a vigilance task. J. Neurosci. 14, 4467-4480.

Bannon, M. J., Reinhard, J. F., Bunney, E. B. Jr., and Roth, R. H. (1982). Unique response to antipsychotic drugs is due to absence of terminal autoreceptors in mesocortical dopamine neurones. Nature 296, 444-446. doi: 10.1038/296444a0

Bari, A., and Robbins, T. W. (2013). Inhibition and impulsivity: behavioral and neural basis of response control. Prog. Neurobiol. 108, 44-79. doi: 10.1016/j.pneurobio.2013.06.005

Beckstead, R. M., Domesick, V. B., and Nauta, W. J. (1979). Efferent connections of the substantia nigra and ventral tegmental area in the rat. Brain Res. 175, 191-217. doi: 10.1016/0006-8993(79)91001-1

Berger, B., Gaspar, P., and Verney, C. (1991). Dopaminergic innervation of the cerebral cortex: unexpected differences between rodents and primates. Trends Neurosci. 14, 21-27. doi: 10.1016/0166-2236(91)90179-X

Berridge, C. W. (2008). Noradrenergic modulation of arousal. Brain Res. Rev. 58, 1-17. doi: 10.1016/j.brainresrev.2007.10.013 
Berridge, C. W., and Waterhouse, B. D. (2003). The locus coeruleus-noradrenergic system: modulation of behavioral state and state-dependent cognitive processes. Brain Res. Brain Res. Rev. 42, 33-84. doi: 10.1016/S0165-0173(03) 00143-7

Bjorklund, A., and Dunnett, S. B. (2007). Dopamine neuron systems in the brain: an update. Trends Neurosci. 30, 194-202. doi: 10.1016/j.tins.2007.03.006

Bouret, S., and Sara, S. J. (2004). Reward expectation, orientation of attention and locus coeruleus-medial frontal cortex interplay during learning. Eur. J. Neurosci. 20, 791-802. doi: 10.1111/j.1460-9568.2004.03526.x

Bouret, S., Ravel, S., and Richmond, B. J. (2012). Complementary neural correlates of motivation in dopaminergic and noradrenergic neurons of monkeys. Front. Behav. Neurosci. 6:40. doi: 10.3389/fnbeh.2012.00040

Bromberg-Martin, E. S., Matsumoto, M., and Hikosaka, O. (2010). Dopamine in motivational control: rewarding, aversive, and alerting. Neuron 68, 815-834. doi: 10.1016/j.neuron.2010.11.022

Chandler, D., and Waterhouse, B. D. (2012). Evidence for broad versus segregated projections from cholinergic and noradrenergic nuclei to functionally and anatomically discrete subregions of prefrontal cortex. Front. Behav. Neurosci. 6:20. doi: 10.3389/fnbeh.2012.00020

Chandler, D. J., Lamperski, C. S., and Waterhouse, B. D. (2013). Identification and distribution of projections from monoaminergic and cholinergic nuclei to functionally differentiated subregions of prefrontal cortex. Brain Res. 1522, 38-58. doi: 10.1016/j.brainres.2013.04.057

Chiodo, L. A., Bannon, M. J., Grace, A. A., Roth, R. H., and Bunney, B. S. (1984). Evidence for the absence of impulse-regulating somatodendritic and synthesis-modulating nerve terminal autoreceptors on subpopulations of mesocortical dopamine neurons. Neuroscience 12, 1-16. doi: 10.1016/0306-4522(84) 90133-7

Curtis, A. L., Pavcovich, L. A., and Valentino, R. J. (1999). Long-term regulation of locus ceruleus sensitivity to corticotropin-releasing factor by swim stress. J. Pharmacol. Exp. Ther. 289, 1211-1219.

Dahlstroem, A., and Fuxe, K. (1964). Evidence for the existence of monoaminecontaining neurons in the central nervous system. I. demonstration of monoamines in the cell bodies of brain stem neurons. Acta Physiol. Scand. Suppl. 62(Suppl. 232), 1-55.

Dalley, J. W., Cardinal, R. N., and Robbins, T. W. (2004). Prefrontal executive and cognitive functions in rodents: neural and neurochemical substrates. Neurosci. Biobehav. Rev. 28, 771-784. doi: 10.1016/j.neubiorev.2004.09.006

Deutch, A. Y., and Roth, R. H. (1990). The determinants of stress-induced activation of the prefrontal cortical dopamine system. Prog. Brain Res. 85, 367-402; discussion 402-403. doi: 10.1016/S0079-6123(08)62691-6

Deutch, A. Y., Goldstein, M., and Roth, R. H. (1986). Activation of the locus coeruleus induced by selective stimulation of the ventral tegmental area. Brain Res. 363, 307-314. doi: 10.1016/0006-8993(86)91016-4

Devilbiss, D. M., and Berridge, C. W. (2006). Low-dose methylphenidate actions on tonic and phasic locus coeruleus discharge. J. Pharmacol. Exp. Ther. 319 1327-1335. doi: 10.1124/jpet.106.110015

Devilbiss, D. M., Page, M. E., and Waterhouse, B. D. (2006). Locus ceruleus regulates sensory encoding by neurons and networks in waking animals. J. Neurosci. 26, 9860-9872. doi: 10.1523/JNEUROSCI.1776-06.2006

Devilbiss, D. M., and Waterhouse, B. D. (2004). The effects of tonic locus ceruleus output on sensory-evoked responses of ventral posterior medial thalamic and barrel field cortical neurons in the awake rat. J. Neurosci. 24, 10773-10785. doi: 10.1523/JNEUROSCI.1573-04.2004

Devilbiss, D. M., Waterhouse, B. D., Berridge, C. W., and Valentino, R. (2012). Corticotropin-releasing factor acting at the locus coeruleus disrupts thalamic and cortical sensory-evoked responses. Neuropsychopharmacology 37, 2020-2030. doi: 10.1038/npp. 2012.50

Driesen, N. R., Leung, H. C., Calhoun, V. D., Constable, R. T., Gueorguieva, R., Hoffman, R., et al. (2008). Impairment of working memory maintenance and response in schizophrenia: functional magnetic resonance imaging evidence. Biol. Psychiatry 64, 1026-1034. doi: 10.1016/j.biopsych.2008.07.029

Drouin, C., Page, M., and Waterhouse, B. (2006). Methylphenidate enhances noradrenergic transmission and suppresses mid- and long-latency sensory responses in the primary somatosensory cortex of awake rats. J. Neurophysiol. 96, 622-632. doi: 10.1152/jn.01310.2005

El Mansari, M., Guiard, B. P., Chernoloz, O., Ghanbari, R., Katz, N., and Blier, P. (2010). Relevance of norepinephrine-dopamine interactions in the treatment of major depressive disorder. CNS Neurosci. Ther. 16, el-e17. doi: 10.1111/j.17555949.2010.00146.x

Foote, S. L., Aston-Jones, G., and Bloom, F. E. (1980). Impulse activity of locus coeruleus neurons in awake rats and monkeys is a function of sensory stimulation and arousal. Proc. Natl. Acad. Sci. U.S.A. 77, 3033-3037. doi: 10.1073/pnas.77.5.3033

Gao, W. J., and Goldman-Rakic, P. S. (2003). Selective modulation of excitatory and inhibitory microcircuits by dopamine. Proc. Natl. Acad. Sci. U.S.A. 100, 2836-2841. doi: 10.1073/pnas.262796399

Gao, W. J., Krimer, L. S., and Goldman-Rakic, P. S. (2001). Presynaptic regulation of recurrent excitation by D1 receptors in prefrontal circuits. Proc. Natl. Acad. Sci. U.S.A. 98, 295-300. doi: 10.1073/pnas.98.1.295

Gao, W. J., Wang, Y., and Goldman-Rakic, P. S. (2003). Dopamine modulation of perisomatic and peridendritic inhibition in prefrontal cortex. J. Neurosci. 23, 1622-1630.

Gesi, M., Soldani, P., and Giorgi, F. S. (2000). A. Santinami, I. Bonaccorsi, and F. Fornai, The role of the locus coeruleus in the development of Parkinson's disease. Neurosci. Biobehav. Rev. 24, 655-668. doi: 10.1016/S0149-7634(00) 00028-2

Goldman-Rakic, P. S. (1994). Working memory dysfunction in schizophrenia. J. Neuropsychiatry Clin. Neurosci. 6, 348-357.

Goldman-Rakic, P. S., and Selemon, L. D. (1997). Functional and anatomical aspects of prefrontal pathology in schizophrenia. Schizophr. Bull. 23, 437-458. doi: $10.1093 /$ schbul/23.3.437

Goldman-Rakic, P. S., Castner, S. A., Svensson, T. H., Siever, L. J., and Williams, G. V. (2004). Targeting the dopamine D1 receptor in schizophrenia: insights for cognitive dysfunction. Psychopharmacology 174, 3-16. doi: 10.1007/s00213-0041793-y

Grace, A. A., Floresco, S. B., Goto, Y., and Lodge, D. J. (2007). Regulation of firing of dopaminergic neurons and control of goal-directed behaviors. Trends Neurosci. 30, 220-227. doi: 10.1016/j.tins.2007.03.003

Grenhoff, J., Nisell, M., Ferre, S., Aston-Jones, G., and Svensson, T. H. (1993). Noradrenergic modulation of midbrain dopamine cell firing elicited by stimulation of the locus coeruleus in the rat. J. Neural Transm. Gen. Sect. 93, 11-25. doi: 10.1007/BF01244934

Grimm, J., Mueller, A., Hefti, F., and Rosenthal, A. (2004). Molecular basis for catecholaminergic neuron diversity. Proc. Natl. Acad. Sci. U.S.A. 101, 1389113896. doi: 10.1073/pnas.0405340101

Grzanna, R., and Molliver, M. E. (1980). The locus coeruleus in the rat: an immunohistochemical delineation. Neuroscience 5, 21-40. doi: 10.1016/03064522(80)90068-8

Haber, S. N., and Fudge, J. L. (1997). The primate substantia nigra and VTA: integrative circuitry and function. Crit. Rev. Neurobiol. 11, 323-342. doi: 10.1615/CritRevNeurobiol.v11.i4.40

Horvitz, J. C. (2000). Mesolimbocortical and nigrostriatal dopamine responses to salient non-reward events. Neuroscience 96, 651-656. doi: 10.1016/S03064522(00)00019-1

Lammel, S., Hetzel, A., Hackel, O., Jones, I., Liss, B., and Roeper, J. (2008). Unique properties of mesoprefrontal neurons within a dual mesocorticolimbic dopamine system. Neuron 57, 760-773. doi: 10.1016/j.neuron.2008.01.022

Lammel, S., Lim, B. K., and Malenka, R. C. (2013). Reward and aversion in a heterogeneous midbrain dopamine system. Neuropharmacology. 46(Part B), 351359. doi: 10.1016/j.neuropharm.2013.03.019

Lammel, S., Lim, B. K., Ran, C., Huang, K. W., Betley, M. J., Tye, K. M., et al. (2012). Input-specific control of reward and aversion in the ventral tegmental area. Nature 491, 212-217. doi: 10.1038/nature11527

Lategan, A. J., Marien, M. R., and Colpaert, F. C. (1990). Effects of locus coeruleus lesions on the release of endogenous dopamine in the rat nucleus accumbens and caudate nucleus as determined by intracerebral microdialysis. Brain Res. 523, 134-138. doi: 10.1016/0006-8993(90)91646-X

Lewis, D. A., Campbell, M. J., Foote, S. L., Goldstein, M., and Morrison, J. H. (1987). The distribution of tyrosine hydroxylase-immunoreactive fibers in primate neocortex is widespread but regionally specific. J. Neurosci. 7, 279-290.

Loughlin, S. E., Foote, S. L., and Bloom, F. E. (1986a). Efferent projections of nucleus locus coeruleus: topographic organization of cells of origin demonstrated by three-dimensional reconstruction. Neuroscience 18, 291-306. doi: 10.1016/03064522(86)90155-7 
Loughlin, S. E., Foote, S. L., and Grzanna, R. (1986b). Efferent projections of nucleus locus coeruleus: morphologic subpopulations have different efferent targets. Neuroscience 18, 307-319. doi: 10.1016/0306-4522(86) 90156-9

Loughlin, S. E., Foote, S. L., and Fallon, J. H. (1982). Locus coeruleus projections to cortex: topography, morphology and collateralization. Brain Res. Bull. 9, 287-294. doi: 10.1016/0361-9230(82)90142-3

Mao, Z. M., Arnsten, A. F., and Li, B. M. (1999). Local infusion of an alpha-1 adrenergic agonist into the prefrontal cortex impairs spatial working memory performance in monkeys. Biol. Psychiatry 46, 1259-1265. doi: 10.1016/S00063223(99)00139-0

McGaughy, J., Newman, L. A., and Darling, J. (2008a). Atomoxetine reverses attentional deficits produced by noradrenergic deafferentation of medial prefrontal cortex. Psychopharmacology 200, 39-50. doi: 10.1007/s00213-0081097-8

McGaughy, J., Ross, R. S., and Eichenbaum, H. (2008b). Noradrenergic, but not cholinergic, deafferentation of prefrontal cortex impairs attentional set-shifting. Neuroscience 153, 63-71. doi: 10.1016/j.neuroscience.2008.01.064

McMillan, P. J., White, S. S., Franklin, A., Greenup, J. L., Leverenz, J. B., Raskind M. A., et al. (2011). Differential response of the central noradrenergic nervous system to the loss of locus coeruleus neurons in Parkinson's disease and Alzheimer's disease. Brain Res. 1373, 240-252. doi: 10.1016/j.brainres.2010. 12.015

Miguelez, C., Grandoso, L., and Ugedo, L. (2011). Locus coeruleus and dorsal raphe neuron activity and response to acute antidepressant administration in a rat model of Parkinson's disease. Int. J. Neuropsychopharmacol. 14, 187-200. doi 10.1017/S146114571000043X

Mingote, S., de Bruin, J. P., and Feenstra, M. G. (2004). Noradrenaline and dopamine efflux in the prefrontal cortex in relation to appetitive classical conditioning. $J$. Neurosci. 24, 2475-2480. doi: 10.1523/JNEUROSCI.4547-03.2004

Morilak, D. A., Barrera, G., Echevarria, D. J., Garcia, A. S., Hernandez, A., Ma, S., et al. (2005). Role of brain norepinephrine in the behavioral response to stress. Prog. Neuropsychopharmacol. Biol. Psychiatry 29, 1214-1224. doi: 10.1016/j.pnpbp.2005.08.007

Morrison, J. H., Grzanna, R., Molliver, M. E., and Coyle, J. T. (1978). The distribution and orientation of noradrenergic fibers in neocortex of the rat: an immunofluorescence study. J. Comp. Neurol. 181, 17-39. doi: 10.1002/cne.901810103

Newman, L. A., Darling, J., and McGaughy, J. (2008). Atomoxetine reverses attentional deficits produced by noradrenergic deafferentation of medial prefrontal cortex. Psychopharmacology (Berl.) 200, 39-50. doi: 10.1007/s00213-0081097-8

Porrino, L. J., and Goldman-Rakic, P. S. (1982). Brainstem innervation of prefrontal and anterior cingulate cortex in the rhesus monkey revealed by retrograde transport of HRP. J. Comp. Neurol. 205, 63-76. doi: 10.1002/cne.9020 50107

Rajkowski, J., Kubiak, P., and Aston-Jones, G. (1994). Locus coeruleus activity in monkey: phasic and tonic changes are associated with altered vigilance. Brain Res. Bull. 35, 607-616. doi: 10.1016/0361-9230(94)90175-9

Robbins, T. W., and Arnsten, A. F. (2009). The neuropsychopharmacology of frontoexecutive function: monoaminergic modulation. Annu. Rev. Neurosci. 32, 267287. doi: 10.1146/annurev.neuro.051508.135535

Robertson, S. D., Plummer, N. W., de Marchena, J., and Jensen, P. (2013). Developmental origins of central norepinephrine neuron diversity. Nat. Neurosci. 16 1016-1023. doi: 10.1038/nn.3458

Roeper, J. (2013). Dissecting the diversity of midbrain dopamine neurons. Trends Neurosci. 36, 336-342. doi: 10.1016/j.tins.2013.03.003

Rossetti, Z. L., and Carboni, S. (2005). Noradrenaline and dopamine elevations in the rat prefrontal cortex in spatial working memory. J. Neurosci. 25, 2322-2329. doi: 10.1523/JNEUROSCI.3038-04.2005

Sara, S. J., and Bouret, S. (2012). Orienting and reorienting: the locus coeruleus mediates cognition through arousal. Neuron 76, 130-141. doi: 10.1016/j.neuron.2012.09.011

Schultz, W. (2007). Multiple dopamine functions at different time courses. Annu. Rev. Neurosci. 30, 259-288. doi: 10.1146/annurev.neuro.28.061604.135722

Seamans, J. K., and Yang, C. R. (2004). The principal features and mechanisms of dopamine modulation in the prefrontal cortex. Prog. Neurobiol. 74, 1-58. doi: 10.1016/j.pneurobio.2004.05.006
Seu, E., Lang, A., Rivera, R. J., and Jentsch, J. D. (2009). Inhibition of the norepinephrine transporter improves behavioral flexibility in rats and monkeys. Psychopharmacology (Berl.) 202, 505-519. doi: 10.1007/s00213-0081250-4

Snyder, K., Wang, W. W., Han, R., McFadden, K., and Valentino, R. J. (2012). Corticotropin-releasing factor in the norepinephrine nucleus, locus coeruleus, facilitates behavioral flexibility. Neuropsychopharmacology 37, 520-530. doi: 10.1038/npp. 2011.218

Stevens, A. A., Goldman-Rakic, P. S., Gore, J. C., Fulbright, R. K., and Wexler, B. E. (1998). Cortical dysfunction in schizophrenia during auditory word and tone working memory demonstrated by functional magnetic resonance imaging. Arch. Gen. Psychiatry 55, 1097-1103. doi: 10.1001/archpsyc.55. 12.1097

Stuber, G. D., Klanker, M., de Ridder, B., Bowers, M. S., Joosten, R. N., Feenstra, M. G., et al. (2008). Reward-predictive cues enhance excitatory synaptic strength onto midbrain dopamine neurons. Science 321, 1690-1692. doi: 10.1126/science. 1160873

Swanson, L. W. (1982). The projections of the ventral tegmental area and adjacent regions: a combined fluorescent retrograde tracer and immunofluorescence study in the rat. Brain Res. Bull. 9, 321-353. doi: 10.1016/0361-9230(82) 90145-9

Szot, P., Miguelez, C., White, S. S., Franklin, A., Sikkema, C., Wilkinson, C. W., et al. (2010). A comprehensive analysis of the effect of DSP4 on the locus coeruleus noradrenergic system in the rat. Neuroscience 166, 279-291. doi: 10.1016/j.neuroscience.2009.12.027

Ungless, M. A., and Grace, A. A. (2012). Are you or aren t you? Challenges associated with physiologically identifying dopamine neurons. Trends Neurosci. 35, 422-430. doi: 10.1016/j.tins.2012.02.003

Valentino, R. J., and Foote, S. L. (1987). Corticotropin-releasing factor disrupts sensory responses of brain noradrenergic neurons. Neuroendocrinology 45, 28-36. doi: $10.1159 / 000124700$

Valentino, R. J., and Foote, S. L. (1988). Corticotropin-releasing hormone increases tonic but not sensory-evoked activity of noradrenergic locus coeruleus neurons in unanesthetized rats. J. Neurosci. 8, 1016-1025.

Volkow, N. D., Wang, G. J., Fowler, J. S., and Tomasi, D. (2012). Addiction circuitry in the human brain. Annu. Rev. Pharmacol. Toxicol. 52, 321-336. doi: 10.1146/annurev-pharmtox-010611-134625

Volkow, N. D., Wang, G. J., Fowler, J. S., Tomasi, D., and Telang, F. (2011). Addiction: beyond dopamine reward circuitry. Proc. Natl. Acad. Sci. U.S.A. 108, 1503715042. doi: 10.1073/pnas.1010654108

Wang, H. X., Waterhouse, B. D., and Gao, W. J. (2013). Selective suppression of excitatory synapses on GABAergic interneurons by norepinephrine in juvenile rat prefrontal cortical microcircuitry. Neuroscience 246, 312-328. doi: 10.1016/j.neuroscience.2013.05.009

Weinshenker, D. (2008). Functional consequences of locus coeruleus degeneration in Alzheimer's disease. Curr. Alzheimer Res. 5, 342-345. doi: 10.2174/156720508784533286

Williams, G. V., and Goldman-Rakic, P. S. (1995). Modulation of memory fields by dopamine D1 receptors in prefrontal cortex. Nature 376, 572-575. doi: $10.1038 / 376572 \mathrm{a} 0$

Conflict of Interest Statement: The authors declare that the research was conducted in the absence of any commercial or financial relationships that could be construed as a potential conflict of interest.

Received: 14 January 2014; accepted: 27 April 2014; published online: 21 May 2014. Citation: Chandler DJ, Waterhouse BD and Gao W-J (2014) New perspectives on catecholaminergic regulation of executive circuits: evidence for independent modulation of prefrontal functions by midbrain dopaminergic and noradrenergic neurons. Front. Neural Circuits 8:53. doi: 10.3389/fncir.2014.00053

This article was submitted to the journal Frontiers in Neural Circuits.

Copyright (c) 2014 Chandler, Waterhouse and Gao. This is an open-access article distributed under the terms of the Creative Commons Attribution License (CC BY). The use, distribution or reproduction in other forums is permitted, provided the original author(s) or licensor are credited and that the original publication in this journal is cited, in accordance with accepted academic practice. No use, distribution or reproduction is permitted which does not comply with these terms. 